\section{Development and validation of a food frequency questionnaire (FFO-Porto Alegre) for adolescent, adult and elderly populations from Southern Brazil}

\author{
Desenvolvimento e validação de um questionário \\ de freqüência alimentar (QFA-Porto Alegre) para \\ a população de adolescentes, adultos e idosos \\ do Sul do Brasil
}

Ruth Liane Henn 1

Sandra Costa Fuchs 2 Leila Beltrami Moreira 2 Flavio Danni Fuchs ${ }^{3}$

\footnotetext{
1 Programa de Pós-graduação em Saúde Coletiva

Universidade do Vale do Rio dos Sinos, São Leopoldo, Brasil.

2 Faculdade de Medicina, Universidade Federal do Rio Grande do Sul, Porto Alegre, Brasil.

${ }^{3}$ Hospital de Clínicas de Porto Alegre, Universidade Federal do Rio Grande do Sul Porto Alegre, Brasil.

Correspondence S. C. Fuchs

Programa de Pós-graduação em Epidemiologia, Faculdade de Medicina, Universidade Federal do Rio Grande do Sul. Rua Ramiro Barcelos 2600, sala 415, Porto Alegre, RS 90035-003, Brasil. scfuchs@terra.com.br
}

\section{Abstract}

This study assessed the validity of a food frequency questionnaire (FFQ-Porto Alegre), covering 135 food items, in comparison with the average of two consecutive 24-hour dietary recall questionnaires for adolescents, adults, and elderly who were randomly selected from a population-based survey. The Pearson correlation coefficients and cross-classification by quartiles of intake were used. The nutrients were log transformed and energy adjusted. The mean of adjusted de-attenuated correlation coefficient for adolescents was 0.44 and ranged from 0.18 (zinc) to 0.69 (folate) and for adult and elderly participants they were, respectively, 0.42 , ranging from 0.16 (iron) to 0.73 (energy) and 0.52 , ranging from 0.25 (vitamin $E$ ) to 0.84 (energy). The average classification percentage into the same or adjacent quartile for the two methods was $74.6 \%$ for adolescents, $74.9 \%$ for adults, and $81.2 \%$ for the elderly population. The FFQ showed fair relative validity for adolescents and adults, and may be used to study the dietary determinants of obesity and non-transmissible diseases in epidemiological surveys.

Food Consumption; Questionnaires; Validation Studies; Aged; Adolescent

\section{Introduction}

The establishment of overall eating patterns - the combination of foods and nutrients consumed by individuals $1-$ makes it possible to identify the determinants of overweight, which are associated with high blood pressure 2 and other non-transmissible diseases ${ }^{3}$. There are several methods for the assessment of food and nutrient consumption and energy intake, including 24-hour recall (24hR), food diary, food frequency questionnaire (FFQ), and biomarkers 4 . All methods have advantages and limitations and the choice among them depends on the purpose for which the information is intended. The $24 \mathrm{hR}$ is based on gathering information on the consumption of drinks and foods in a period of 24 hours, through the interview. In the food diary, all foods and beverages consumed in one or more days are recorded by the participant. On the other hand, the FFQ is based on the frequency of consumption of each item from a list of foods, for a period of time ranging from months to a year. It is based on the premise that the ingestion of food for a longer period of time is more appropriate when it comes to assessing the association between dietary patterns and chronic diseases. The $24 \mathrm{hR}$ and the FFQ are the most frequently used tools in epidemiological studies nowadays 4,5 .

The need to develop and to validate FFQs for the population where it will be used is widely recognized ${ }^{4}$, even though this has not often been 
done and described in an appropriate manner. The FFQ development requires several decisions about food selection, the number of items to be investigated, and the method for measuring the portion sizes, if the FFQ is quantitative. Validation is usually performed using a food diary 6 or $24 \mathrm{hR} 7,8,9$ as the reference method. In general, the validity is determined through correlation coefficients, comparing the average intake, obtained via the FFQ and the reference method, as well as the classification of individuals according to the nutrient intake distribution in both methods 10 .

In this study, we described the development and validation of a FFQ in two populations - adolescents and adults - living in Porto Alegre, the capital of Rio Grande do Sul State in Brazil, who were randomly selected to participate in a population-based study (SOFT Study - Syndrome of Obesity and Risk Factors for Cardiovascular Diseases).

\section{Methods}

\section{FFO development}

In order to identify food items that should be part of the FFQ, 268 individuals between the ages of 12 and 90 were selected at schools, universities, and recreational areas for the elderly population, in Porto Alegre and the Metropolitan area. The sample comprised 61 adolescents (14.9 \pm 1.3 years), 120 adults ( $30.2 \pm 10.9$ years) and 87 elderly (68.7 \pm 7.5 years) who completed a $24 \mathrm{hR}$. The reported food items were compared to those identified in a population-based study, conducted in Rio de Janeiro, Brazil 11. The list of foods and preparations reported in Porto Alegre was similar to that of Rio de Janeiro. However, fourteen food items that were part of FFQ in Rio de Janeiro - for instance, angu, okra, yam, cará, oxtail - did not take part in the food list that was developed for the FFQ-Porto Alegre. Another 64 food items were added including nuts (walnuts, almonds, chestnuts), polenta, lentils, sushi, sashimi, tofu, etc. The decision was initially based upon the frequency of consumption ( $5 \%$ or more), but exceptions included food items that represent the influence of German, Italian, Japanese, and southern Brazilian cuisine. This food list was tested again and some changes were made, particularly in the amount of food items of the FFQ, with a reduction in the number of items through its aggregation. The inclusion of options related to the frequency, quantity and portions of the foods was pre-tested in groups of persons with different ages and education levels. The final version of the FFQ was pre-tested and contained 135 food items. Figure 1 shows the Portuguese version of the FFQ.

\section{FFQ validation}

The SOFT Study investigated adolescents (1219) and adults (20-90 years of age) using a crosssectional design, from a population-based sampling from Porto Alegre. Details of the study can be found elsewhere 12 . In this validity study, a sub-sample of 127 adolescents and 127 adults was selected through a simple random sampling based on the previous household mapping. The sample size was based on the recommendation that 100-200 subjects should be interviewed 5 . Participants were interviewed at home by nutritionists and graduate students, certified to apply the instruments, and participants responded at the same interview to two $24 \mathrm{hR}$ and one FFQ. The 24hR investigated the food intake of two consecutive periods of 24 hours prior to the interview and the FFQ surveyed the food intake of the last 12 months. The application of the two dietary surveys was randomized in order to minimize potential bias increasing the awareness and affecting the answers given in the second method.

In the FFQ, the information on food intake was converted into daily intake (grams or milliliters). In the $24 \mathrm{hR}$, all food items, preparation methods, recipes, quantity and size of portions were recorded, as well as the brand of industrialized products. The portion size was documented from the album of photographs of different foods and household items, usually employed for their measurement 13. Quality control procedures included the food survey supervision, and repeated interviews, with additional questioning if needed. The nutritional analysis of food intake in the FFQ and $24 \mathrm{hR}$ was performed using the Programa de Apoio à Nutrição, developed by the Center for Health Informatics at the Federal University in São Paulo. Some food items that were not part of the database of the program were added based on the Table for Evaluation of Food Consumption in Household Measures 14 and the food labels.

\section{Other variables under study}

For the analysis in the validity study, age (categorized into 12-14 and 15-19 years old for adolescents, 20-59 years old for adults, and 60-90 years old for the elderly), sex, weight, and height were recorded in a standardized questionnaire, in addition to food consumption. Body mass index (BMI, $\mathrm{kg} / \mathrm{m}^{2}$ ) was calculated as the ratio between weight (in kilograms) and height (in meters, squared). Overweight was defined as BMI greater than or equal to $25 \mathrm{~kg} / \mathrm{m}^{2}$, according to the recommendations of the World Health Organization (WHO) 15. For adolescents, BMI for age and sex was used to determine overweight, based on the 
Figure 1

Food frequency questionnaire used in the Syndrome of Obesity and Risk Factors for Cardiovascular Diseases (SOFT Study).

Do <MÊS> do ano passado até agora, quantas vezes por dia ou por semana ou por mês ou por ano você comeu os alimentos que eu vou citar? Quantos meses do ano? Quantas <PORÇÕES> você comeu a cada vez?

\begin{tabular}{|c|c|c|c|c|c|c|c|c|c|c|c|c|c|c|c|c|c|c|}
\hline \multirow[t]{2}{*}{ Alimentos } & \multicolumn{12}{|c|}{ Quantas vezes } & \multicolumn{5}{|c|}{ Unidade de tempo } & \multirow[t]{2}{*}{ Quantidade } \\
\hline & 0 & 1 & 2 & 3 & 4 & 5 & 6 & 7 & 8 & 9 & 10 & Outro & D & $S$ & M & A & Meses/Ano & \\
\hline Cacetinho/Bisnaguinha & & & & & & & & & & & & & & & & & & ( ) UP ( ) UG \\
\hline $\begin{array}{l}\text { Sanduíche de presunto e queijo/ } \\
\text { Torrada }\end{array}$ & & & & & & & & & & & & & & & & & & ( ) Unidade \\
\hline $\begin{array}{l}\text { Pão (sanduíche/forma/leite/caseiro/ } \\
\text { manteiga/batata) }\end{array}$ & & & & & & & & & & & & & & & & & & ( ) Fatia \\
\hline Pão (integral/centeio/trigo/aveia) & & & & & & & & & & & & & & & & & & ( ) Fatia \\
\hline Pão light & & & & & & & & & & & & & & & & & & ( ) Fatia \\
\hline Sanduíche natural & & & & & & & & & & & & & & & & & & ( ) Unidade \\
\hline Cuca/Pão doce & & & & & & & & & & & & & & & & & & () FP () FM () FG \\
\hline Bolo & & & & & & & & & & & & & & & & & & () FP () FM () FG \\
\hline Pão de queijo & & & & & & & & & & & & & & & & & & ( ) UP () UM () UG \\
\hline Bolacha (doce/recheada) & & & & & & & & & & & & & & & & & & () Unidade () Pacote \\
\hline Bolacha salgada & & & & & & & & & & & & & & & & & & ( ) Unidade () Pacote \\
\hline Sucrilhos & & & & & & & & & & & & & & & & & & () $1 / 2$ PS () PS () XP () XM () XG \\
\hline Aveia/Germe de trigo/Granola & & & & & & & & & & & & & & & & & & ( ) CSopa \\
\hline Barra de cereal & & & & & & & & & & & & & & & & & & ( ) Unidade \\
\hline Nescau, Toddy ou outros & & & & & & & & & & & & & & & & & & ( ) CChá ( ) CSopa \\
\hline Milk shakes/Batida & & & & & & & & & & & & & & & & & & () $\mathrm{CP}$ () $\mathrm{CM}$ () $\mathrm{CG}$ \\
\hline Leite integral & & & & & & & & & & & & & & & & & & ( ) $\mathrm{CP}$ () $\mathrm{CM}$ () $\mathrm{CG}$ \\
\hline Leite desnatado & & & & & & & & & & & & & & & & & & () $\mathrm{CP}$ () $\mathrm{CM}$ () $\mathrm{CG}$ \\
\hline Leite semi-desnatado & & & & & & & & & & & & & & & & & & () $\mathrm{CP}$ () $\mathrm{CM}$ () $\mathrm{CG}$ \\
\hline Leite de soja & & & & & & & & & & & & & & & & & & ( ) $\mathrm{CP}$ () $\mathrm{CM}$ () $\mathrm{CG}$ \\
\hline logurte integral & & & & & & & & & & & & & & & & & & ( ) Pote () GP () GG \\
\hline logurte (desnatado/light) & & & & & & & & & & & & & & & & & & ( ) Pote () GP () GG \\
\hline Requeijão normal/Käshimier & & & & & & & & & & & & & & & & & & () Ponta de faca () CChá \\
\hline Requeijão light & & & & & & & & & & & & & & & & & & () Ponta de faca () CChá \\
\hline $\begin{array}{l}\text { Queijo (mussarela/lanche/colonial/ } \\
\text { provolone) }\end{array}$ & & & & & & & & & & & & & & & & & & ( ) FP () FM () FG \\
\hline Queijo (branco/minas/ricota) & & & & & & & & & & & & & & & & & & () FP () FM () FG \\
\hline Creme de leite/Nata & & & & & & & & & & & & & & & & & & ( ) CChá () CSopa \\
\hline Leite condensado & & & & & & & & & & & & & & & & & & ( ) CChá () CSopa \\
\hline Manteiga/Margarina normal & & & & & & & & & & & & & & & & & & () Ponta de faca () CChá \\
\hline Margarina light & & & & & & & & & & & & & & & & & & () Ponta de faca () CChá \\
\hline Maionese normal & & & & & & & & & & & & & & & & & & ( ) Ponta de faca () CChá \\
\hline Maionese light & & & & & & & & & & & & & & & & & & () Ponta de faca () CChá \\
\hline $\begin{array}{l}\text { Mortadela/Salame/Murcilha/Presunto } \\
\text { gordo }\end{array}$ & & & & & & & & & & & & & & & & & & () FP () FM () FG \\
\hline $\begin{array}{l}\text { Presunto magro/Peito de peru/ } \\
\text { Chester }\end{array}$ & & & & & & & & & & & & & & & & & & () FP () FM () FG \\
\hline $\begin{array}{l}\text { Mel/Geléia/Chimia/Uvada/Goiabada/ } \\
\text { Figada/Pessegada/Marmelada }\end{array}$ & & & & & & & & & & & & & & & & & & () Ponta de faca () CChá \\
\hline Geléia diet/Chimia diet & & & & & & & & & & & & & & & & & & ( ) CChá ( ) CSopa \\
\hline Salada de frutas & & & & & & & & & & & & & & & & & & ( ) CP () CM () CG () Pote \\
\hline
\end{tabular}

(continues) 
Figure 1 (continued)

Do <MÊS> do ano passado até agora, quantas vezes por dia ou por semana ou por mês ou por ano você comeu os alimentos que eu vou citar Quantos meses do ano? Quantas <PORÇÕES> você comeu a cada vez?

\begin{tabular}{|c|c|c|c|c|c|c|c|c|c|c|c|c|c|c|c|c|c|c|}
\hline \multirow[t]{2}{*}{ Alimentos } & \multicolumn{12}{|c|}{ Quantas vezes } & \multicolumn{5}{|c|}{ Unidade de tempo } & \multirow[t]{2}{*}{ Quantidade } \\
\hline & 0 & 1 & 2 & 3 & 4 & 5 & 6 & 7 & 8 & 9 & 10 & Outro & D & $\mathrm{S}$ & M & A & Meses/Ano & \\
\hline Abacate & & & & & & & & & & & & & & & & & & ( ) UP ( ) UG \\
\hline Abacaxi & & & & & & & & & & & & & & & & & & ( ) FP () FG \\
\hline Banana & & & & & & & & & & & & & & & & & & () UP () UM () UG \\
\hline Caqui & & & & & & & & & & & & & & & & & & ( ) UP ( ) UG \\
\hline Laranja/Bergamota & & & & & & & & & & & & & & & & & & ( ) UP () UG \\
\hline Maçã/Pêra & & & & & & & & & & & & & & & & & & ( ) UP () UG \\
\hline Mamão/Papaia & & & & & & & & & & & & & & & & & & () Fatia () Unidade \\
\hline Melancia & & & & & & & & & & & & & & & & & & () FP () FM () FG \\
\hline Melão & & & & & & & & & & & & & & & & & & () FP () FM () FG \\
\hline Morango & & & & & & & & & & & & & & & & & & ( ) UP () UG \\
\hline Pêssego/Ameixa & & & & & & & & & & & & & & & & & & ( ) UP () UG \\
\hline Uva & & & & & & & & & & & & & & & & & & () $\mathrm{CaP}$ () $\mathrm{CaM}$ () $\mathrm{CaG}$ \\
\hline Suco de laranja & & & & & & & & & & & & & & & & & & () $\mathrm{CP}$ () $\mathrm{CM}$ () $\mathrm{CG}$ \\
\hline Suco de frutas natural & & & & & & & & & & & & & & & & & & () $\mathrm{CP}$ () $\mathrm{CM}$ () $\mathrm{CG}$ \\
\hline Sucos artificiais adoçados & & & & & & & & & & & & & & & & & & () $\mathrm{CP}$ () $\mathrm{CM}$ () $\mathrm{CG}$ \\
\hline Arroz branco & & & & & & & & & & & & & & & & & & ( ) CSopa \\
\hline Arroz integral & & & & & & & & & & & & & & & & & & ( ) CSopa \\
\hline Feijão/Lentilha & & & & & & & & & & & & & & & & & & () $\mathrm{CoP}$ ( ) CoM () CoG \\
\hline Grão de bico & & & & & & & & & & & & & & & & & & ( ) CoP () CoM () CoG \\
\hline Canjica & & & & & & & & & & & & & & & & & & () $\mathrm{CoP}$ () $\mathrm{CoM}$ () CoG \\
\hline Salada de batata ou maionese & & & & & & & & & & & & & & & & & & ( ) CSopa \\
\hline Batata cozida & & & & & & & & & & & & & & & & & & () UP () UM () UG \\
\hline Nhoque & & & & & & & & & & & & & & & & & & () CSopa () Pegador \\
\hline Batata frita & & & & & & & & & & & & & & & & & & ( ) Pegador () Porção \\
\hline Aipim cozido & & & & & & & & & & & & & & & & & & () PP () PM () PG \\
\hline Aipim frito/Polenta frita & & & & & & & & & & & & & & & & & & () PP () PM () PG \\
\hline Polenta (cozida/assada) & & & & & & & & & & & & & & & & & & () CSopa () PP () PM () PG \\
\hline Bolinho de arroz ou batata & & & & & & & & & & & & & & & & & & () Unidade \\
\hline Macarrão/Massas & & & & & & & & & & & & & & & & & & ( ) Pegador () CSopa \\
\hline Panqueca/Canelone/Rondele & & & & & & & & & & & & & & & & & & ( ) Unidade \\
\hline Lasanha & & & & & & & & & & & & & & & & & & () PP () PM () PG \\
\hline Abóbora/Moranga & & & & & & & & & & & & & & & & & & ( ) CSopa \\
\hline Abobrinha/Chuchu & & & & & & & & & & & & & & & & & & ( ) CSopa \\
\hline $\begin{array}{l}\text { Agrião/Alface/Chicória/Radiche/ } \\
\text { Rúcula }\end{array}$ & & & & & & & & & & & & & & & & & & () Pires () Folhas \\
\hline Salada misturada & & & & & & & & & & & & & & & & & & ( ) CSopa () Pires \\
\hline Beterraba (crua/cozida) & & & & & & & & & & & & & & & & & & () CSopa \\
\hline Brócolis/Couve/Espinafre & & & & & & & & & & & & & & & & & & () CSopa () Ramo \\
\hline Cebola (crua/assada) & & & & & & & & & & & & & & & & & & () CSopa \\
\hline Cenoura (crua/cozida) & & & & & & & & & & & & & & & & & & () CSopa \\
\hline Couve-flor & & & & & & & & & & & & & & & & & & () CSopa () Ramo \\
\hline Milho verde & & & & & & & & & & & & & & & & & & () EspigaP ( ) EspigaM \\
\hline Milho enlatado & & & & & & & & & & & & & & & & & & () CSopa \\
\hline Repolho & & & & & & & & & & & & & & & & & & () CSopa \\
\hline Vagem & & & & & & & & & & & & & & & & & & ( ) CSopa \\
\hline
\end{tabular}

(continues) 
Do <MÊS> do ano passado até agora, quantas vezes por dia ou por semana ou por mês ou por ano você comeu os alimentos que eu vou citar? Quantos meses do ano? Quantas <PORÇÕES> você comeu a cada vez?

\begin{tabular}{|c|c|c|c|c|c|c|c|c|c|c|c|c|c|c|c|c|c|c|}
\hline \multirow[t]{2}{*}{ Alimentos } & \multicolumn{12}{|c|}{ Quantas vezes } & \multicolumn{5}{|c|}{ Unidade de tempo } & \multirow[t]{2}{*}{ Quantidade } \\
\hline & 0 & 1 & 2 & 3 & 4 & 5 & 6 & 7 & 8 & 9 & 10 & Outro & $\mathrm{D}$ & $\mathbf{S}$ & $M$ & A & Meses/Ano & \\
\hline Tomate cru & & & & & & & & & & & & & & & & & & ( ) UP () UM () UG \\
\hline Legumes variados & & & & & & & & & & & & & & & & & & ( ) CSopa \\
\hline Legumes empanados fritos & & & & & & & & & & & & & & & & & & () Ramo () Rodela \\
\hline Sopa de legumes ou de verduras & & & & & & & & & & & & & & & & & & () $\operatorname{CoP}$ () $\operatorname{CoM}$ () CoG \\
\hline Sopa com arroz/massa/capeletti & & & & & & & & & & & & & & & & & & () $\operatorname{CoP}$ () CoM () CoG \\
\hline Ovo/Omelete/Ovo mexido & & & & & & & & & & & & & & & & & & ( ) Unidade () CSopa \\
\hline $\begin{array}{l}\text { Cachorro-quente/Xis de carne ou } \\
\text { frango }\end{array}$ & & & & & & & & & & & & & & & & & & ( ) Unidade \\
\hline Pastelão/Empadão/Quiche & & & & & & & & & & & & & & & & & & ( ) PP ( ) PM ( ) PG \\
\hline Pizza & & & & & & & & & & & & & & & & & & () FP () FM () FG \\
\hline $\begin{array}{l}\text { Pastel/Coxinha/Rissoles/Croquete } \\
\text { (fritos) }\end{array}$ & & & & & & & & & & & & & & & & & & ( ) UP () UM () UG \\
\hline Guisado/Almôndega & & & & & & & & & & & & & & & & & & () CSopa () Unidade \\
\hline Churrasco & & & & & & & & & & & & & & & & & & () PP () PM ()PG \\
\hline Carne de gado & & & & & & & & & & & & & & & & & & () PP ()PM ()PG \\
\hline Frango com pele & & & & & & & & & & & & & & & & & & () PP () PM () PG \\
\hline Frango sem pele & & & & & & & & & & & & & & & & & & () PP ()PM ()PG \\
\hline Carne de porco & & & & & & & & & & & & & & & & & & ( ) PP ( ) PM () PG \\
\hline Carne de soja & & & & & & & & & & & & & & & & & & () CSopa \\
\hline Bucho/Mondongo & & & & & & & & & & & & & & & & & & ( ) CSopa () Prato \\
\hline Vísceras (moela/fígado) & & & & & & & & & & & & & & & & & & () Pedaço ( ) CSopa \\
\hline Coraçãozinho & & & & & & & & & & & & & & & & & & () Unidade \\
\hline Bacon/Toucinho & & & & & & & & & & & & & & & & & & Registrar só a freqüência \\
\hline Lingüiça/Salsichão & & & & & & & & & & & & & & & & & & ( ) Unidade () CSopa \\
\hline Salsicha & & & & & & & & & & & & & & & & & & ( ) UP ( ) UM ( ) UG \\
\hline Peixe (fresco/congelado) & & & & & & & & & & & & & & & & & & ( ) PP () PM () PG \\
\hline Tofu & & & & & & & & & & & & & & & & & & ( ) Fatia \\
\hline Sushi & & & & & & & & & & & & & & & & & & ( ) Unidade \\
\hline Sashimi & & & & & & & & & & & & & & & & & & ( ) Fatia \\
\hline Sardinha/Atum (conserva) & & & & & & & & & & & & & & & & & & ( ) Lata ( ) CSopa \\
\hline Camarão & & & & & & & & & & & & & & & & & & () CSopa () Unidade \\
\hline Chocolate em barra/Bombom & & & & & & & & & & & & & & & & & & ( ) UP ( ) UM () UG \\
\hline $\begin{array}{l}\text { Brigadeiro/Negrinho/Doce com } \\
\text { chocolate }\end{array}$ & & & & & & & & & & & & & & & & & & ( ) Unidade \\
\hline $\begin{array}{l}\text { Pudim/Ambrosia/Doce de leite/Arroz } \\
\text { doce/Flan }\end{array}$ & & & & & & & & & & & & & & & & & & ( ) CSopa () PP () PM () PG \\
\hline Sorvete & & & & & & & & & & & & & & & & & & ( ) CSopa () Bola \\
\hline Sorvete light & & & & & & & & & & & & & & & & & & ( ) CSopa () Bola \\
\hline Tortas em geral & & & & & & & & & & & & & & & & & & () PP () PM () PG \\
\hline Fruta em calda & & & & & & & & & & & & & & & & & & ( ) PP ( ) PM () PG \\
\hline Café preto passado & & & & & & & & & & & & & & & & & & ( ) XP( ()XM() XG \\
\hline Café expresso & & & & & & & & & & & & & & & & & & ( ) $X P() \times M() X G$ \\
\hline Café solúvel & & & & & & & & & & & & & & & & & & ( ) CChá \\
\hline Café cappuccino & & & & & & & & & & & & & & & & & & ( ) $X P() \times M() \times G$ \\
\hline Café sem cafeína & & & & & & & & & & & & & & & & & & () XP()XM ()XG \\
\hline
\end{tabular}


Do <MÊS> do ano passado até agora, quantas vezes por dia ou por semana ou por mês ou por ano você comeu os alimentos que eu vou citar? Quantos meses do ano? Quantas <PORÇÕES> você comeu a cada vez?

\begin{tabular}{|c|c|c|c|c|c|c|c|c|c|c|c|c|c|c|c|c|c|c|}
\hline \multirow[t]{2}{*}{ Alimentos } & \multicolumn{12}{|c|}{ Quantas vezes } & \multicolumn{5}{|c|}{ Unidade de tempo } & \multirow[t]{2}{*}{ Quantidade } \\
\hline & 0 & 1 & 2 & 3 & 4 & 5 & 6 & 7 & 8 & 9 & 10 & Outro & D & S & M & A & Meses/Ano & \\
\hline Chá & & & & & & & & & & & & & & & & & & () XP () XM () XG \\
\hline Chimarrão & & & & & & & & & & & & & & & & & & () Cuia () Térmica \\
\hline Água (fora café/chá) & & & & & & & & & & & & & & & & & & () $\mathrm{CP}$ () $\mathrm{CM}$ () $\mathrm{CG}$ \\
\hline Refrigerante & & & & & & & & & & & & & & & & & & () $\mathrm{CP}$ () $\mathrm{CM}$ () $\mathrm{CG}$ \\
\hline Refrigerante (diet/light) & & & & & & & & & & & & & & & & & & () $\mathrm{CP}$ () $\mathrm{CM}$ () $\mathrm{CG}$ \\
\hline Açúcar & & & & & & & & & & & & & & & & & & ( ) CChá ( ) CSopa \\
\hline Adoçante (líquido/pó) & & & & & & & & & & & & & & & & & & () Gotas () Sachês \\
\hline $\begin{array}{l}\text { Amendoim/Nozes/Castanha-do-Pará/ } \\
\text { Castanha de caju }\end{array}$ & & & & & & & & & & & & & & & & & & () Punhado () Unidade \\
\hline Uva passa & & & & & & & & & & & & & & & & & & ( ) CSopa \\
\hline $\begin{array}{l}\text { Guloseimas/Paçoquinha/ } \\
\text { Rapadurinha/Maria-mole/ } \\
\text { Merenguinho/Puxa-puxa }\end{array}$ & & & & & & & & & & & & & & & & & & ( ) Unidade \\
\hline Bala/Chiclete & & & & & & & & & & & & & & & & & & ( ) Unidade \\
\hline Pipoca & & & & & & & & & & & & & & & & & & () $\mathrm{SaP}$ () SaM () SaG \\
\hline Chips/Fandango/Milhopã & & & & & & & & & & & & & & & & & & () SaP () SaM () SaG \\
\hline Outro & & & & & & & & & & & & & & & & & & \\
\hline
\end{tabular}

CaP: cacho pequeno; CaM: cacho médio; CaG: cacho grande; CChá: colher de chá; CSopa: colher de sopa; CoP: concha pequena; CoM: concha média; CoG: concha grande; CP: copo pequeno; CM: copo médio; CG: copo grande; EspigaP: espiga pequena; EspigaM: espiga média; FP: fatia pequena; FM: fatia média; FG: fatia grande; GP: garrafa pequena; GG: garrafa grande; PP: pedaço pequeno; PM: pedaço médio; PG: pedaço grande; PS: prato de sopa; UP: unidade pequena; UM: unidade média; UG: unidade grande; SaP: saco pequeno; SaM: saco médio; SaG: saco grande; XP: xícara pequena; XM: xícara média; XG: xícara grande.

International Obesity Task Force (IOTF) guidelines, corresponding to overweight (BMI $\geq 25 \mathrm{~kg}$ / $\left.\mathrm{m}^{2}\right)$ at the age of 18 years 16 .

\section{Data analysis}

Each food item in the FFQ and $24 \mathrm{hR}$ was measured as a continuous variable, multiplying the number of times that each item was consumed by its frequency (daily, weekly, monthly, or annual) and by the number of months per year that the food was consumed. The amount was based on the number of portions in predetermined sizes, whether in natural units, household measures, or weights and volumes of usually consumed portions 14 . The consumption of nutrients and energy was transformed by natural logarithm to obtain normal distributions, through the formula $\log (\mathrm{x}+1) 17$.

Mean \pm standard deviation and ratio of the average consumption of nutrients and energy were determined for the FFQ and for the average of two consecutive 24hR. Differences between methods were tested through a paired t test for log-transformed data. In order to assess the relative validity between the FFQ and the average of the two 24hR, Pearson correlations were calculated for non-adjusted nutrients and nutrients corrected for energy. Energy-adjusted nutrient intakes were computed as the residuals from the linear regression model with total caloric intake as the independent variable and absolute nutrient intake as the dependent variable ${ }^{4}$. As the residuals vary from person to person and may have negative values, a constant was added to them. The constant for each nutrient was calculated as the nutrient intake for the average energy intake of the sample ${ }^{4}$. As daily variations in the intra-individual food intake could attenuate the correlations between the FFQ and the reference method, the correlation coefficients were corrected by the ratio of the intra- and inter-individ- 
ual variances, obtained by analysis of variance of a classification criterion in both recalls 4 , through the following equation:

$r_{\nu}=r_{o}(1+\lambda / n)^{1 / 2}$;

where $r_{\nu}$ is the true correlation, $r_{o}$ is the correlation observed between the FFQ and average of the $24 \mathrm{hR}, \lambda$ is the intra- and inter-individual variance ratio in the $24 \mathrm{hR}$, and $n$ is the number of replicates, which comprised two recalls. The correlation coefficients between 0.4 and 0.7 indicate a good concordance between both diet evaluation methods 4 .

The concordance between the FFQ and average of the $24 \mathrm{hR}$ was assessed via classification of individuals according to their distribution in energy and nutrient quartiles. The exact concordance percentage (classification in the same quartile by both methods), classification in the same quartile or an adjacent quartile, and disagreement (classification in opposite quartiles) were calculated. SPSS version 14 (SPSS Inc., Chicago, USA) was used for data analysis.

\section{Results}

From a total of 127 participants randomly selected for the validity study, 13 declined to participate, representing $10.2 \%$ of refusals, and one participant was excluded from the analysis for being on a diet, for a diagnostic procedure. Among the 127 randomly selected adolescents (12-19 years of age), 125 provided complete data for the validity study. Among 113 adults aged 55 \pm 18 years, $42 \%$ were 60 years old or older, there was a predominance of women, and $46.9 \%$ were overweight (Table 1). In the adolescent population, $56 \%$ were girls, had an average $15 \pm 2$ years of age and $10.4 \%$ were overweight.

Table 2 shows statistically significant differences between the estimated consumption from the FFQ and the average of the two $24 \mathrm{hR}$ for adult and elderly participants. The exception was the protein intake among teenagers, the average consumption of which was similar $(p=0.5)$. The absolute intake of macro- and micronutrients detected by the FFQ was higher than that of the $24 \mathrm{hR}$, particularly for fiber, vitamins A, E, C for adults and elderly participants, as the ratio of consumption in the FFQ was about twice that in the $24 \mathrm{hR}$.

The not adjusted correlation coefficient between the two methods of food consumption investigation - FFQ and 24hR - ranged from 0.31 (vitamin E) to 0.57 (calcium) among adolescents (Table 3), 0.29 (vitamin E) to 0.66 (calcium) among adults, and 0.34 (vitamin E) to 0.69 (vitamin C) among the elderly. The adjusted and de-attenuated correlation coefficients for most nutrients were higher than 0.50 for elderly participants, yet only five and four of them had coefficients equal to or higher than 0.50 for adults and adolescents, respectively. Overall, the energy adjustment increased the magnitude of correlation coefficients, except for fiber and vitamins A and $\mathrm{C}$ among the elderly, and calcium for adolescents. There was an increase in the de-attenuated and energy-adjusted coefficients for most nutri-

Table 1

Characteristics of participants in the validity study of the food frequency questionnaire (FFQ) among adolescents and adults in the Syndrome of Obesity and Risk Factors for Cardiovascular Diseases (SOFT Study).

\begin{tabular}{|c|c|c|c|}
\hline Adolescents & Boys $(n=55)$ & Girls $(n=70)$ & Total \\
\hline \multicolumn{4}{|l|}{ Age (years) } \\
\hline $12-14[n(\%)]$ & $26(47.0)$ & $36(51.0)$ & $62(49.6)$ \\
\hline $15-19[\mathrm{n}(\%)]$ & $29(53.0)$ & $34(49.0)$ & $63(50.4)$ \\
\hline Overweight * [\% (95\%Cl)] & $5.5(1.0-11.6)$ & $14.3(5.9-22.3)$ & $10.4(5.0-15.8)$ \\
\hline Adults & $\operatorname{Men}(n=40)$ & Women $(n=73)$ & Total \\
\hline \multicolumn{4}{|l|}{ Age (years) } \\
\hline $20-59[\mathrm{n}(\%)]$ & $25(62.0)$ & $41(56.0)$ & $66(58.4)$ \\
\hline $60-90[n(\%)]$ & $15(38.0)$ & $32(44.0)$ & $47(41.6)$ \\
\hline Overweight ** [\% (95\%Cl)] & $42.5(26.5-58.5)$ & $47.9(37.6-61.1)$ & $46.9(37.6-56.2)$ \\
\hline
\end{tabular}

* $\mathrm{BMI}$ by age and sex, corresponding to $\mathrm{BMI} \geq 25 \mathrm{~kg} / \mathrm{m}^{2}$ at 18 years old;

$\star \star B M I \geq 25 \mathrm{~kg} / \mathrm{m}^{2}$ 
Average intake of energy, macronutrients and micronutrients estimated by the food frequency questionnaire (FFQ), 24-hour recall (24hR) survey, and the ratio between both methods in adolescents, adults $<60$ years and adults $\geq 60$ years in the Syndrome of Obesity and Risk Factors for Cardiovascular Diseases (SOFT Study).

\begin{tabular}{|c|c|c|c|c|c|c|c|c|c|}
\hline \multirow[t]{2}{*}{ Nutrients } & \multicolumn{3}{|c|}{ Adolescents $(n=125)$} & \multicolumn{3}{|c|}{ Adults $(n=66)$} & \multicolumn{3}{|c|}{ Elderly $(n=47)$} \\
\hline & $\begin{array}{c}\text { FFQ } \\
\text { Average } \\
( \pm \mathrm{SD})\end{array}$ & $\begin{array}{c}24 \mathrm{hR} \\
\text { Average } \\
( \pm \mathrm{SD})\end{array}$ & $\begin{array}{l}\text { FFQ/ } \\
24 \mathrm{hR} \\
\text { ratio }\end{array}$ & $\begin{array}{c}\text { FFQ } \\
\text { Average } \\
( \pm S D)\end{array}$ & $\begin{array}{c}24 \mathrm{hR} \\
\text { Average } \\
( \pm \mathrm{SD})\end{array}$ & $\begin{array}{l}\text { FFQ/ } \\
24 \mathrm{hR} \\
\text { ratio }\end{array}$ & $\begin{array}{c}\text { FFQ } \\
\text { Average } \\
( \pm \mathrm{SD})\end{array}$ & $\begin{array}{c}24 \mathrm{hR} \\
\text { Average } \\
( \pm \mathrm{SD})\end{array}$ & $\begin{array}{l}\text { FFQ/ } \\
24 \mathrm{hR} \\
\text { ratio }\end{array}$ \\
\hline Energy (kcal) & $\begin{array}{c}2,376.7 \\
(894.4) *\end{array}$ & $\begin{array}{l}2,105.8 \\
(892.3)\end{array}$ & 1.1 & $\begin{array}{c}2476.3 \\
(888.1)^{*}\end{array}$ & $\begin{array}{l}1979.2 \\
(807.5)\end{array}$ & 1.3 & $\begin{array}{l}1,894.4 \\
(781.0)\end{array}$ & $\begin{array}{c}1528.7 \\
(728.4)^{*}\end{array}$ & 1.2 \\
\hline Carbohydrates (g) & $\begin{array}{c}323.7 \\
(122.0) \text { * }\end{array}$ & $\begin{array}{c}288.5 \\
(121.2)\end{array}$ & 1.1 & $\begin{array}{c}305.3 \\
(116.6) \text { * }\end{array}$ & $\begin{array}{c}247.7 \\
(102.4)\end{array}$ & 1.2 & $\begin{array}{c}248.4 \\
(112.5)\end{array}$ & $\begin{array}{c}202.2 \\
(106.1) \text { * }\end{array}$ & 1.2 \\
\hline Protein (g) & $\begin{array}{c}71.4 \\
(26.2)\end{array}$ & $\begin{array}{c}72.5 \\
(38.7)\end{array}$ & 1.0 & $\begin{array}{c}95.3 \\
(38.1) \text { * }\end{array}$ & $\begin{array}{c}77.7 \\
(36.8)\end{array}$ & 1.2 & $\begin{array}{c}71.8 \\
(28.1)\end{array}$ & $\begin{array}{c}63.7 \\
(36.0) \text { * }\end{array}$ & 1.1 \\
\hline Fat (g) & $\begin{array}{c}98.2 \\
(41.1) \text { * }\end{array}$ & $\begin{array}{c}76.9 \\
(39.3)\end{array}$ & 1.3 & $\begin{array}{c}99.8 \\
(41.9) \text { * }\end{array}$ & $\begin{array}{c}73.6 \\
(35.3)\end{array}$ & 1.4 & $\begin{array}{c}72.7 \\
(32.6)\end{array}$ & $\begin{array}{c}53.1 \\
(26.4) \text { * }\end{array}$ & 1.4 \\
\hline Saturated fat (g) & $\begin{array}{c}30.2 \\
(12.6) \text { * }\end{array}$ & $\begin{array}{c}21.7 \\
(13.0)\end{array}$ & 1.4 & $\begin{array}{c}30.8 \\
(14.4) \text { * }\end{array}$ & $\begin{array}{c}21.1 \\
(14.1)\end{array}$ & 1.5 & $\begin{array}{c}23.4 \\
(11.4)\end{array}$ & $\begin{array}{c}15.5 \\
(9.5) \text { * }\end{array}$ & 1.5 \\
\hline Cholesterol (mg) & $\begin{array}{c}218.9 \\
(118.6) *\end{array}$ & $\begin{array}{c}178.3 \\
(129.0)\end{array}$ & 1.2 & $\begin{array}{c}278.4 \\
(139.4) \text { * }\end{array}$ & $\begin{array}{c}214.3 \\
(146.3)\end{array}$ & 1.3 & $\begin{array}{c}218.7 \\
(153.5)\end{array}$ & $\begin{array}{c}181.8 \\
(157.0) \text { * }\end{array}$ & 1.2 \\
\hline Fiber (g) & $19.7(7.6)$ * & $\begin{array}{l}14.1 \\
(8.4)\end{array}$ & 1.4 & $\begin{array}{c}21.9 \\
(8.7) \text { * }\end{array}$ & $\begin{array}{c}9.8 \\
(5.1)\end{array}$ & 2.2 & $\begin{array}{c}20.3 \\
(10.8)\end{array}$ & $\begin{array}{c}10.3 \\
(6.7) \text { * }\end{array}$ & 2.0 \\
\hline Calcium (mg) & $\begin{array}{c}880.7 \\
(387.3) \text { * }\end{array}$ & $\begin{array}{c}634.7 \\
(386.6)\end{array}$ & 1.4 & $\begin{array}{c}852.1 \\
(497.4) \text { * }\end{array}$ & $\begin{array}{c}634.2 \\
(451.5)\end{array}$ & 1.3 & $\begin{array}{c}844.5 \\
(393.0)\end{array}$ & $\begin{array}{c}622.4 \\
(355.2) \text { * }\end{array}$ & 1.4 \\
\hline Iron (mg) & $\begin{array}{c}15.7 \\
(6.0) \text { * }\end{array}$ & $13.0(5.9)$ & 1.2 & $\begin{array}{c}17.0 \\
(7.6) *\end{array}$ & $\begin{array}{l}12.9 \\
(5.7)\end{array}$ & 1.3 & $13.5(7.9)$ & $\begin{array}{c}10.5 \\
(5.5) \text { * }\end{array}$ & 1.3 \\
\hline Zinc (mg) & $\begin{array}{c}8.6 \\
(3.4) \text { * }\end{array}$ & $7.0(4.4)$ & 1.2 & $\begin{array}{c}12.5 \\
(6.0) \text { * }\end{array}$ & $\begin{array}{c}9.6 \\
(6.6)\end{array}$ & 1.3 & $9.3(3.8)$ & $\begin{array}{c}7.2 \\
(3.9) \text { * }\end{array}$ & 1.3 \\
\hline $\begin{array}{l}\text { Vitamin A (equivalent } \\
\text { to retinol) }\end{array}$ & $\begin{array}{c}867.9 \\
(593.5) *\end{array}$ & $\begin{array}{c}649.7 \\
(1179.0)\end{array}$ & 1.3 & $\begin{array}{l}1,289.9 \\
(816.8)\end{array}$ & $\begin{array}{c}678.8 \\
(1372.2)\end{array}$ & 1.9 & $\begin{array}{l}1,189.9 \\
(739.0)\end{array}$ & $\begin{array}{c}669.5 \\
(851.4) *\end{array}$ & 1.8 \\
\hline $\begin{array}{l}\text { Vitamin E (equivalent } \\
\text { to alpha-tocopherol) }\end{array}$ & $\begin{array}{c}15.2 \\
(6.0) *\end{array}$ & $9.2(7.0)$ & 1.7 & $\begin{array}{c}17.0 \\
(7.1) \star \star\end{array}$ & $\begin{array}{r}9.6 \\
(6.2)\end{array}$ & 1.8 & $13.7(6.1)$ & $\begin{array}{c}7.4 \\
(3.4) \text { * }\end{array}$ & 1.8 \\
\hline Vitamin C (mg) & $\begin{array}{c}116.8 \\
(68.0) *\end{array}$ & $\begin{array}{c}133.6 \\
(558.0)\end{array}$ & 0.9 & $\begin{array}{c}151.30 \\
(98.0) \star \star \star\end{array}$ & $\begin{array}{c}74.1 \\
(62.2)\end{array}$ & 2.0 & $\begin{array}{c}165.8 \\
(119.0)\end{array}$ & $\begin{array}{c}86.0 \\
(77.5) *\end{array}$ & 1.9 \\
\hline Folate (mcg) & $\begin{array}{c}235.7 \\
(99.7) \text { * }\end{array}$ & $\begin{array}{c}151.7 \\
(105.1)\end{array}$ & 1.6 & $\begin{array}{c}288.9 \\
(119.3) *\end{array}$ & $\begin{array}{c}192.5 \\
(111.9)\end{array}$ & 1.5 & $\begin{array}{c}257.0 \\
(123.3)\end{array}$ & $\begin{array}{c}172.3 \\
(127.3) *\end{array}$ & 1.5 \\
\hline
\end{tabular}

* Significantly different from the average of the two 24hR: paired t test for logarithmically transformed data (natural log); $p<0.001$;

** Significantly different from the average of the two 24hR: paired t test for logarithmically transformed data (natural log); $p=0.018$;

$\star \star \star$ Significantly different from the average of the two 24hR: paired t test for logarithmically transformed data (natural log); $p=0.002$.

ents, but vitamin $\mathrm{E}$ for adults and cholesterol for adolescents. The average de-attenuated energy correlation coefficients for adults $(\mathrm{r}=0.42)$ and adolescents $(r=0.44)$ were similar (Table 3$)$, but lower than for the elderly $(r=0.52)$.

Table 4 shows that the agreement between methods for food intake in the same quartile or in an adjacent quartile was $69 \%$ or more for all the nutrients, except fat and zinc among adolescents, above $70 \%$ for most nutrients, except vitamin $\mathrm{E}$, iron, zinc, and folate among adults, and for the elderly greater than 0,72 for all nutrients except carbohydrates. On average, $75 \%$ of the adults and adolescents and $81 \%$ of the elderly were classified in the same or in an adjacent quartile by both methods. Considering the error in the classification of consumption quartiles, an average of $6.2 \%$ of adolescents and $6.1 \%$ of adults were in opposite quartiles versus $3 \%$ of the elderly.

\section{Discussion}

In this study we described the development of a FFQ with 135 food items, providing details for the assessment of its suitability as well as for replica- 
Table 3

Correlation coefficients between the food frequency questionnaire (FFO) and the average of both 24-hour recall (24hR) surveys for adults and adolescents in the Syndrome of Obesity and Risk Factors for Cardiovascular Diseases (SOFT Study) $(n=238)$ *.

\begin{tabular}{|c|c|c|c|c|c|c|c|c|c|}
\hline \multirow[t]{2}{*}{ Nutrients } & \multicolumn{3}{|c|}{ Adolescents $(n=125)$} & \multicolumn{3}{|c|}{ Adults $(n=66)$} & \multicolumn{3}{|c|}{ Elderly $(n=47)$} \\
\hline & $\begin{array}{c}\text { Not } \\
\text { adjusted }\end{array}$ & $\begin{array}{l}\text { Adjusted } \\
\text { for } \\
\text { energy ** }\end{array}$ & $\begin{array}{c}\text { Adjusted } \\
\text { and de- } \\
\text { attenuated } \star \star \star\end{array}$ & $\begin{array}{c}\text { Not } \\
\text { adjusted }\end{array}$ & $\begin{array}{l}\text { Adjusted } \\
\text { for } \\
\text { energy ** }\end{array}$ & $\begin{array}{l}\text { Adjusted } \\
\text { and de- } \\
\text { attenuated } \\
\quad \star \star \star\end{array}$ & $\begin{array}{c}\text { Not } \\
\text { adjusted }\end{array}$ & $\begin{array}{l}\text { Adjusted } \\
\text { for } \\
\text { energy ** }\end{array}$ & $\begin{array}{c}\text { Adjusted } \\
\text { and de- } \\
\text { attenuated } \star \star \star\end{array}$ \\
\hline Energy (kcal) & 0.49 & - & $0.64 \#$ & 0.60 & - & $0.73 \#$ & 0.76 & - & $0.84 \#$ \\
\hline Carbohydrate (g) & 0.53 & 0.26 & 0.32 & 0.56 & 0.45 & 0.53 & 0.67 & 0.39 & 0.43 \\
\hline Protein (g) & 0.54 & 0.31 & 0.41 & 0.58 & 0.42 & 0.55 & 0.73 & 0.46 & 0.51 \\
\hline Fat (g) & 0.43 & 0.26 & 0.34 & 0.59 & 0.42 & 0.54 & 0.68 & 0.47 & 0.55 \\
\hline Saturated fat (g) & 0.39 & 0.32 & 0.34 & 0.64 & 0.36 & 0.47 & 0.67 & 0.50 & 0.58 \\
\hline Cholesterol (mg) & 0.46 & 0.30 & 0.30 & 0.58 & 0.51 & 0.64 & 0.60 & 0.55 & 0.67 \\
\hline Fiber (g) & 0.46 & 0.40 & 0.57 & 0.44 & 0.33 & 0.40 & 0.46 & 0.48 & 0.51 \\
\hline Calcium (mg) & 0.57 & 0.62 & 0.73 & 0.66 & 0.56 & 0.63 & 0.62 & 0.58 & 0.64 \\
\hline Iron (mg) & 0.48 & 0.33 & 0.45 & 0.49 & 0.13 & 0.16 & 0.51 & 0.28 & 0.31 \\
\hline Zinc (mg) & 0.35 & 0.10 & 0.18 & 0.44 & 0.17 & 0.25 & 0.60 & 0.38 & 0.45 \\
\hline $\begin{array}{l}\text { Vitamin A } \\
\text { (equivalent to } \\
\text { retinol) }\end{array}$ & 0.40 & 0.36 & 0.41 & 0.21 & 0.16 & 0.26 & 0.44 & 0.46 & 0.61 \\
\hline $\begin{array}{l}\text { Vitamin E } \\
\text { (equivalent to } \\
\text { alpha-tocopherol) }\end{array}$ & 0.31 & 0.16 & 0.21 & 0.29 & 0.06 & 0.07 & 0.34 & 0.22 & 0.25 \\
\hline Vitamin C (mg) & 0.50 & 0.49 & 0.55 & 0.37 & 0.35 & 0.47 & 0.69 & 0.70 & 0.77 \\
\hline Folate (mcg) & 0.45 & 0.43 & 0.69 & 0.41 & 0.19 & 0.24 & 0.46 & 0.32 & 0.36 \\
\hline Average & 0.45 & 0.32 & 0.44 & 0.49 & 0.32 & 0.42 & 0.59 & 0.45 & 0.52 \\
\hline
\end{tabular}

* Analysis performed with logarithmically transformed nutrients (natural log);

** Adjusted for energy according to the residual method;

$\star \star \star$ Corrected for the intra-individual variation in both 24 -hour recall $(24 \mathrm{hR})$;

\# Only de-attenuated.

tion elsewhere. Thereafter we present the validation of this questionnaire by comparing it with the average of two $24 \mathrm{hR}$. There was a satisfactory global agreement for energy, macronutrients, and micronutrients. However, the energy and nutrient consumption was higher in the FFQ than in the $24 \mathrm{hR}$. This disparity was previously described for a validity study conducted in a population of French adults and adolescents 18 . A similar result was also detected among Greek adolescents, even though the FFQ was compared with the average of three $24 \mathrm{hR}$ 19. In South Asian immigrants living in the United Kingdom, the absolute energy and nutrient intake estimated by a FFQ was higher than in $24 \mathrm{hR}$, yet an extreme variation for some nutrients was identified, such as $71 \%$ for vitamin $\mathrm{C}$ and $10 \%$ for vitamin B12 20 .

The FFQ may be biased by the list of food items used. The number of items necessary to establish a dietary pattern is a matter of judgment, since a low number may underestimate and a high number may overestimate consumption. The higher consumption of fiber and vitamins by the adult and elderly population, detected in our study, could be explained by the number of fruits and vegetables investigated in the FFQ 4. An alternative explanation for the differences in favor of the FFQ is the predetermined size of the portions used to quantify the food intake, which may differ from those reported using an album of photographs in $24 \mathrm{hR} 21$. On the other hand, there are reports showing higher estimates in the reference method 6 , other presenting equivalence between the methods 7 , or variable directions depending on the type of nutrient 8 . In addition, the period to which subjects report the consumption, two days in the 24-hour recall and one year in the FFQ, is a potential source of bias for food items not consumed on a daily basis.

The $24 \mathrm{hR}$ is widely used as a reference method in food frequency questionnaire validation studies, but their sources of error may not be 
Quartile food consumption classification. comparing the food frequency questionnaire (FFQ) and the 24-hour recall (24hR) survey in adults and adolescents in the Syndrome of Obesity and Risk Factors for Cardiovascular Diseases (SOFT Study) $(n=238)$ *

\begin{tabular}{|c|c|c|c|c|c|c|c|c|c|}
\hline \multirow[t]{2}{*}{ Nutrients } & \multicolumn{3}{|c|}{ Adolescents $(n=125)$} & \multicolumn{3}{|c|}{ Adults $(n=66)$} & \multicolumn{3}{|c|}{ Elderly $(n=47)$} \\
\hline & $\begin{array}{c}\text { Same } \\
\text { quartile }\end{array}$ & $\begin{array}{c}\text { Same } \\
\text { quartile or } \\
\text { adjacent } \\
\text { quartile }\end{array}$ & $\begin{array}{l}\text { Opposed } \\
\text { quartiles }\end{array}$ & $\begin{array}{c}\text { Same } \\
\text { quartile }\end{array}$ & $\begin{array}{c}\text { Same } \\
\text { quartile or } \\
\text { adjacent } \\
\text { quartile }\end{array}$ & $\begin{array}{l}\text { Opposed } \\
\text { quartiles }\end{array}$ & $\begin{array}{c}\text { Same } \\
\text { quartile }\end{array}$ & $\begin{array}{c}\text { Same } \\
\text { quartile or } \\
\text { adjacent } \\
\text { quartile }\end{array}$ & $\begin{array}{l}\text { Opposed } \\
\text { quartiles }\end{array}$ \\
\hline Energy (kcal) ** & 34.4 & 83.2 & 2.4 & 37.9 & 89.4 & 3.0 & 48.9 & 95.7 & 0.0 \\
\hline Carbohydrates (g) & 28.8 & 73.6 & 5.6 & 42.4 & 84.8 & 3.0 & 36.2 & 68.1 & 2.1 \\
\hline Protein (g) & 34.4 & 69.6 & 9.6 & 40.9 & 74.2 & 3.0 & 38.3 & 74.5 & 2.1 \\
\hline Fat (g) & 29.6 & 66.4 & 6.4 & 42.4 & 81.8 & 3.0 & 36.2 & 78.7 & 4.3 \\
\hline Saturated fat (g) & 37.6 & 76.8 & 7.2 & 34.8 & 75.8 & 4.5 & 42.6 & 85.1 & 4.3 \\
\hline Cholesterol (mg) & 31.2 & 72.8 & 5.6 & 30.3 & 71.2 & 4.5 & 48.9 & 89.4 & 2.1 \\
\hline Fiber (g) & 33.6 & 77.6 & 7.2 & 33.3 & 77.3 & 7.6 & 42.6 & 87.2 & 2.1 \\
\hline Calcium (mg) & 50.4 & 83.2 & 0.8 & 30.3 & 81.8 & 3.0 & 44.7 & 72.3 & 6.4 \\
\hline Iron (mg) & 33.6 & 68.8 & 4.0 & 33.3 & 63.6 & 9.1 & 40.4 & 83.0 & 4.3 \\
\hline Zinc (mg) & 27.2 & 63.2 & 10.4 & 24.2 & 69.7 & 9.1 & 27.7 & 76.6 & 2.1 \\
\hline $\begin{array}{l}\text { Vitamin A } \\
\text { (equivalent to } \\
\text { retinol) }\end{array}$ & 33.6 & 77.6 & 8.0 & 34.8 & 71.2 & 9.1 & 23.4 & 80.9 & 2.1 \\
\hline $\begin{array}{l}\text { Vitamin E } \\
\text { (equivalent to } \\
\text { alpha-tocopherol) }\end{array}$ & 33.6 & 68.8 & 8.8 & 27.3 & 65.2 & 10.6 & 27.7 & 72.3 & 6.4 \\
\hline Vitamin C (mg) & 36.0 & 84.0 & 4.8 & 36.4 & 75.8 & 6.1 & 42.6 & 91.5 & 2.1 \\
\hline Folate (mcg) & 39.2 & 79.2 & 5.6 & 27.3 & 66.7 & 9.1 & 40.4 & 80.9 & 2.1 \\
\hline Average & 34.5 & 74.6 & 6.2 & 34.0 & 74.9 & 6.1 & 38.6 & 81.2 & 3.0 \\
\hline
\end{tabular}

* Log values transformed and adjusted for energy via the residue method;

** Only log transformed.

independent, since both methods are based on memory ${ }^{4}$. The specific weekday investigated by the 24hR may have influenced the estimation of nutrient intake, since, in Southern Brazil, people usually have a barbecue on Sundays, which probably accounts for a higher consumption of meat compared to other days of the week.

Another potential limitation is derived from the application of both $24 \mathrm{hR}$ in the same day of the FFQ, which could result in higher correlation coefficients between methods. Sichieri \& Everhart 7, using a similar methodology, but with application of the FFQ followed by the recall of two $24 \mathrm{hR}$ within a space of two weeks, found similar averages of energy consumption fairly correlated $(r=0.44)$. The $24 \mathrm{hR}$ of two consecutive days reduces the within-person variation, and, as many as seven days of data collection should be required for some nutrients and food groups to be correctly assessed 21 . However, it is unrealistic in most large population-based samples and research and statistical modeling may di- minish the limitation of having only a few days of intake 4 .

The values reported here for the energy-adjusted correlation coefficients were higher than the values reported from previous studies 7 . The energy adjustment resulted nevertheless in a reduction in correlation coefficients for most nutrients, confirming previous findings 8,22 . The total energy adjustment was performed on the assumption that each participant reported similar nutrient intakes in both questionnaires 4 . The daily intra-individual food consumption variation, quantified by two $24 \mathrm{hR}$, was corrected with the de-attenuation of the FFQ correlations with the reference method 4 . The increasing of coefficients de-attenuated and corrected for energy indicates the daily intake variability, especially regarding fiber, calcium, folate, vitamins $\mathrm{A}$ and $\mathrm{C}$ for the adolescents; cholesterol for adults; and fiber, calcium, vitamin A and $\mathrm{C}$ for the elderly population. The correlation coefficients adjusted for energy and de-attenuated were higher than 
0.40 for eight of the 14 items assessed, among adolescents and adults, and 11 among the elderly. The correlations observed were within the limits of variation considered acceptable (0.4-0.7) for calibrations studies of diet 4,10 .

Although direct comparisons with other studies are difficult due to differences in the FFQ, reference methods and population characteristics, the magnitude of the correlations was similar to those corrected for energy and de-attenuated, described in other validation studies, both for elderly 23, adults 10 and adolescents 24,25. The correct classification of individuals by the relative amount of nutrient intake is indispensable to explore the association between dietary patterns and risk of disease. In this study, the average proportions of adults and adolescents rightly classified were similar to the findings reported in other studies $6,8,24,25$. Considering that $75 \%$ of adults and adolescents and $81 \%$ of elderly participants were classified in the same or in adjacent quartiles by both methods, it is possible to infer the validity of the FFQ 25,26. Food consumption of participants up to 90 years of age has a potential for bias due to attention and memory decline with age, and might be the reason for the paucity of studies in the elderly. However, the interviews were conducted face-to-face, lasted longer than those answered by adults, and discrepancies in responses were clarified with an adult living at the same house, as a strategy for collecting dietary data from elderly people 27 . Even though the sample was relatively small, it was representative of the target population.

In conclusion, we demonstrated that the FFQ-Porto Alegre has a fair relative validity in adolescents and adults, including large spectrum of participant's age, from 12 to 90 years old. The FFQ-Porto Alegre may be used to study the dietary determinants of obesity and non-transmissible diseases in epidemiological surveys.

\section{Resumo}

Esse estudo avaliou a validade relativa de questionário de freqüência alimentar (QFA-Porto Alegre), com 135 itens, comparando-o à média de dois recordatórios de 24 horas, entre adolescentes, adultos e indivíduos idosos selecionados aleatoriamente da população. Utilizou-se classificação em quartis de ingestão pelos dois métodos e coeficiente de correlação de Pearson para analisar nutrientes transformados em logaritmo natural e ajustados pela energia. O coeficiente de correlação ajustado de-atenuado médio entre adolescentes foi 0,44 e variou de 0,18 (zinco) a 0,69 (folato), em adultos e idosos, respectivamente, foi 0,42 variando de 0,16 (ferro) a 0,73 (energia) e 0,52 variando de 0,25 (vitamina E) a 0,84 (energia). O percentual médio de classificação no mesmo quartil ou adjacente pelos dois métodos foi 74,6\% para adolescentes, 74,9\% para adultos, e 81,2\% para idosos, com classificação média geral de 75\%. O QFA mostrou validade relativa satisfatória para adolescentes e adultos e pode ser usado para analisar a associação entre padrão de dieta e doenças não transmissíveis.

Consumo de Alimentos; Questionários; Estudos de Validação; Idoso; Adolescente

\section{Contributors}

R. L. Henn, S. C. Fuchs, L. B. Moreira and F. D. Fuchs designed the study, supervised the data collection, analyzed and interpreted the data, and wrote the manuscript.

\section{Acknowledgments}

This study was partially funded by the National Research Council (CNPq), including a grant from the Brazilian National Program for Centers of Excellence (PRONEX), Rio Grande do Sul State Research Foundation (FAPERGS; researchers in charge: S. C. Fuchs, L. B. Moreira, and F. D. Fuchs), and the Fundo de Incentivo à Pesquisa e Eventos at the Hospital de Clínicas in Porto Alegre (FIPE/HCPA). 


\section{References}

1. Hu F, editor. Obesity epidemiology. New York: Oxford University Press; 2008.

2. Paeratakul S, Lovejoy J, Ryan D, Brain G. The relation of gender, race and socioeconomic status to obesity and obesity comorbidities in sample of US adults. Int J Obes 2002; 26:1205-10.

3. World Health Organization. Reducing risks, promoting healthy life. Geneva: World Health Organization; 2002.

4. Willett WC. Nutritional epidemiology. Oxford: Oxford University Press; 1998.

5. Lima FEL, Latorre MRDO, Costa MJC, Fisberg RM. Diet and cancer in Northeast Brazil: evaluation of eating habits and food group consumption in relation to breast cancer. Cad Saúde Pública 2008; 24:820-8.

6. Cardoso MA, Kida AA, Tomita LY, Stocco PR. Reproducibility and validity of a food frequency questionnaire among women of Japanese ancestry living in Brazil. Nutr Res 2001; 21:725-33.

7. Sichieri R, Everhart JE. Validity of a Brazilian food frequency questionnaire against dietary recalls and estimated energy intake. Nutr Res 1998; 18:1649-59.

8. Slater B, Philippi ST, Fisberg RM, Latorre MRDO. Validation of a semi-quantitative adolescent food frequency questionnaire applied at a public school in São Paulo, Brazil. Eur J Clin Nutr 2003; 57:62935 .

9. Zanolla AF, Olinto MTA, Henn RL, Wahrlich V, Anjos LA. Avaliação de reprodutibilidade e validade de um questionário de freqüência alimentar em adultos residentes em Porto Alegre, Rio Grande do Sul, Brasil. Cad Saúde Pública 2009; 25:840-8.

10. Cade J, Thompson R, Burley V, Warm D. Development, validation and utilisation of food-frequency questionnaires: a review. Public Health Nutr 2002; 5:567-87.

11. Sichieri R. Epidemiologia da obesidade. Rio de Janeiro: EdUERJ; 1998.

12. Fuchs SC, Moreira LB, Camey AS, Moreira MB, Fuchs FD. Clustering of risk factors for cardiovascular disease among women in Southern Brazil: a population-based study. Cad Saúde Pública 2008; 24 Suppl 2:S285-93.

13. Vitolo MR. Nutrição da gestação ao envelhecimento. Rio de Janeiro: Editora Rubio; 2008.

14. Pinheiro ABV, Lacerda EMA, Benzecry EH, Gomes MCS, Costa VM. Tabela para avaliação de consumo alimentar em medidas caseiras. Rio de Janeiro: Editora Atheneu; 2005.

15. World Health Organization. Report of WHO Consultation in Obesity. Obesity: preventing and managing the global epidemic. Geneva: World Health Organization; 1998.
16. Cole TJ, Bellizzi MC, Flegal KM, Dietz WH. Establishing a standard definition for child overweight and obesity worldwide: international survey. BMJ 2000; 320:1240-3.

17. Chen Y, Ahsan H, Parvez F, Howe GR. Validity of a food-frequency questionnaire for a large prospective cohort study in Bangladesh. Br J Nutr 2004; 92:851-9.

18. Deschamps V, de Lauzon-Guillain B, Lafay L, Borys JM, Charles MA, Romon M. Reproducibility and relative validity of a food-frequency questionnaire among French adults and adolescents. Eur J Clin Nutr 2009; 63:282-91.

19. Papadopoulou SK, Barboukis V, Dalkiranis A, Hassapidou M, Petridou A, Mougios V. Validation of a questionnaire assessing food frequency and nutritional intake in Greek adolescents. Int J Food Sci Nutr 2008; 59:148-54.

20. Sevak L, Mangtani P, McCormack V, Bhakta D, Kassam-Khamis T, Silva IS. Validation of a food frequency questionnaire to assess macro- and micronutrient intake among South Asians in the United Kingdom. Eur J Nutr 2004; 43:160-8.

21. Hartwell DL, Henry CJK. Comparison of a selfadministered quantitative food amount frequency questionnaire with 4-day estimated food records. Int J Food Sci Nutr 2001; 52:151-9.

22. Fornés NS, Stringhini MLF, Elias BM. Reproducibility and validity of a food-frequency questionnaire among low-income Brazilian workers. Public Health Nutr 2003; 6:821-7.

23. Shahar D, Fraser D, Shai I, Vardi H. Development of a Food Frequency Questionnaire (FFQ) for an elderly population based on a population survey. J Nutr 2003; 133:3625-9.

24. Johansson I, Hallmans G, Wikman A, Biessy C, Riboli E, Kaaks R. Validation and calibration of food-frequency questionnaire measurements in the Northern Sweden Health and Disease cohort. Public Health Nutr 2002; 5:487-96.

25. Rockett HR, Breitenbach M, Frazier AL, Witschi J, Wolf AM, Field AE, et al. Validation of a youth/adolescent food frequency questionnaire. Prev Med 1997; 26:808-16.

26. Masson LF, McNeill G, Tomany JO, Simpson JA, Peace HS, Wei L, et al. Statistical approaches for assessing the relative validity of a food-frequency questionnaire: use of correlation coefficients and the kappa statistic. Public Health Nutr 2003; 6: 313-21.

27. Ervin RB, Smiciklas-Wright H. Using encoding and retrieval strategies to improve 24-hour dietary recalls among older adults. J Am Diet Assoc 1998; 98:989-94.

Submitted on 19/Aug/2009

Final version resubmitted on 26/Nov/2009

Approved on 05/Feb/2010 